\section{BRAZIULAN JOURNAL \\ OF MEDICAL AND BIOLOGICAL RESEARCH}

www.bjournal.com.br
ISSN 1414-431X

Volume 45 (12) 1102-1340 December 2012

\section{BIOMIDICAL SCIENCES}

AND

CLINICAL INVESTIGATION

Braz J Med Biol Res, December 2012, Volume 45(12) 1320-1326

doi: 10.1590/S0100-879X2012007500156

Psychiatric disorders and cardiac anxiety in exercising and sedentary coronary artery disease patients: a case-control study

A. Sardinha, C.G.S. Araújo and A.E. Nardi

The Brazilian Journal of Medical and Biological Research is partially financed by

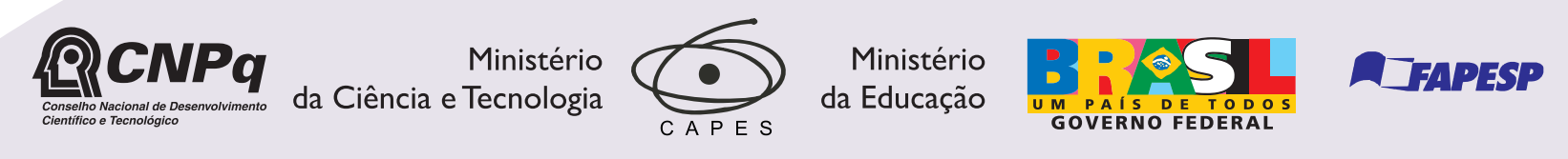

Institutional Sponsors

SciE/
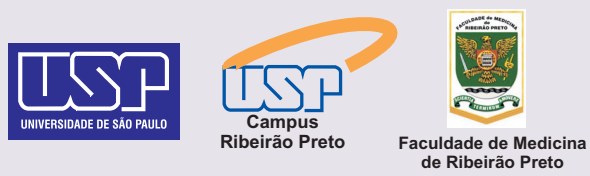

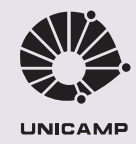

UNICAMP $\oplus$ SHIMADZU

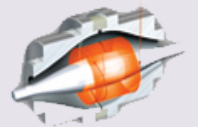

1DID Associaçăo Explore High - Performance MS Orbitrap Technology In Proteomics \& Metabolomics analitica $\underset{\text { analiticaweb.com.br }}{\text { Thermo }}$ S I N TIFIC 


\title{
Psychiatric disorders and cardiac anxiety in exercising and sedentary coronary artery disease patients: a case-control study
}

\author{
A. Sardinha ${ }^{1}$, C.G.S. Araújo ${ }^{2}$ and A.E. Nardi ${ }^{1}$ \\ ${ }^{1}$ Laboratório de Pânico e Respiração, Instituto de Psiquiatria, \\ Universidade Federal do Rio de Janeiro, Rio de Janeiro, RJ, Brasil \\ 2Programa de Pós-Graduação em Ciências do Exercício e do Esporte, \\ Universidade Gama Filho, Rio de Janeiro, RJ, Brasil
}

\begin{abstract}
Regular physical exercise has been shown to favorably influence mood and anxiety; however, there are few studies regarding psychiatric aspects of physically active patients with coronary artery disease (CAD). The objective of the present study was to compare the prevalence of psychiatric disorders and cardiac anxiety in sedentary and exercising CAD patients. A total sample of 119 CAD patients (74 men) were enrolled in a case-control study. The subjects were interviewed to identify psychiatric disorders and responded to the Cardiac Anxiety Questionnaire. In the exercise group $(N=60)$, there was a lower prevalence (45 vs $81 \% ; \mathrm{P}<0.001$ ) of at least one psychiatric diagnosis, as well as multiple comorbidities, when compared to the sedentary group $(\mathrm{N}=59)$. Considering the Cardiac Anxiety Questionnaire, sedentary patients presented higher scores compared to exercisers (mean $\pm \mathrm{SEM}=55.8 \pm 1.9$ vs $37.3 \pm 1.6 ; \mathrm{P}<0.001$ ). In a regression model, to be attending a medically supervised exercise program presented a relevant potential for a $35 \%$ reduction in cardiac anxiety. CAD patients regularly attending an exercise program presented less current psychiatric diagnoses and multiple mental-related comorbidities and lower scores of cardiac anxiety. These salutary mental effects add to the already known health benefits of exercise for CAD patients.
\end{abstract}

Key words: Exercise training; Mental health; Depression; Cardiovascular disease; Cardiac rehabilitation

\section{Introduction}

Coronary artery disease (CAD) is the leading cause of death and disability in highly industrialized countries (1). The literature supports clinically important associations between psychiatric illness and chronic medical conditions such as CAD. Depression is currently considered to be a risk factor for CAD and is also known to adversely affect self-care and increase the risk of complications and mortality (2). Although most of the available studies have focused on the impact of depression in CAD patients, recent evidence indicates that anxiety and hostility are associated with a higher number of negative cardiac events (3).

Although there are fewer studies regarding anxiety disorders compared to depression in CAD patients, robust epidemiological and clinical evidence shows that they might play an equally important role. Anxiety has been found to independently increase the risk of developing CAD in healthy subjects (4), lead to worsening of existing CAD and also impact treatment outcomes negatively, as anxious patients can be less likely to adhere to prescribed medical treatments (5). The rates of major adverse coronary events, including recurrent ischemic events and death, in patients with CAD are significantly increased in patients with depression as well as in the presence of anxiety (6). In addition, health care costs are higher and health-related quality of life is lower in anxious (7) and in depressed patients with CAD (8). In this respect, anxiety disorders in medically ill patients should not be ignored and should be considered jointly with depression when developing strategies for screening and intervention (2).

Health-related anxiety (HRA) is a specific type of anxiety that leads to increased worrying about one's health and the belief that normal bodily symptoms are threatening, harmful and medically serious, despite evidence to the contrary. Hypochondriasis represents an intense form

Correspondence: A. Sardinha, Laboratório de Pânico e Respiração, IPUB/UFRJ, Av. Venceslau Braz, 71, $22290-140$ Rio de Janeiro, RJ, Brasil. E-mail: alinesardinhapsi@gmail.com

Received June 21, 2012. Accepted August 8, 2012. Available online September 28, 2012. Published December 17, 2012. 
of HRA (9). Cardiac anxiety (10), on the other hand, is a particular presentation of HRA that refers to the fear of cardiac-related stimuli and sensations based upon their perceived negative consequences. Because cardiac-related events are perceived to be aversive and dangerous, this behavior can promote anxiety that may elicit undesirable body symptoms (11).

Regular physical exercise has been shown to play an important role in mental health maintenance (12). The prevalence and incidence of depression and anxiety are lower in those who are regularly active, while being physically active also seems to reduce symptoms of depression and anxiety (13). In this respect, exercise training is a healthy behavior with a minimal risk of adverse events that could be an effective and practical tool for reducing anxiety among patients (3). Bearing in mind that exercising could be useful in the treatment of psychological problems in CAD patients, the present study aimed to compare the prevalence of psychiatric comorbidities and the level of cardiac anxiety in sedentary and exercising CAD patients.

\section{Material and Methods}

A case-control study was conducted on a sample of 119 CAD patients recruited from two different institutions, who will be referred to hereafter as exercise $(N=60)$ and sedentary $(\mathrm{N}=59)$ groups. Both groups presented similar demographic characteristics, differing specifically by the fact that the exercise group was composed of CAD patients participating in a supervised exercise program while the sedentary group consisted of CAD patients from a typical outpatient clinic with no formal exercise program.

\section{Exercise group}

The exercise group consisted of 60 CAD patients attending a medically supervised exercise program. The CAD patients were referred by their attending cardiologist and enrolled in the supervised program with no limit in the duration of participation. Briefly, the exercise session consisted of individualized prescribed $30 \mathrm{~min}$ of treadmill and/ or upper and lower limbs cyclergometer aerobic exercises, followed by $20-30 \mathrm{~min}$ of strengthening and stretching exercises. Data were collected before and after one of the exercise sessions in a separate area within the clinic in order to preserve confidentiality. All patients had a formal diagnosis of CAD, documented by a history of myocardial infarction or coronary artery revascularization procedures or by the presence of significant obstructive coronary lesions as evidenced by angiography. Inclusion criteria were agreement to participate, regular compliance (attendance of over $70 \%$ of prescribed sessions) with the supervised exercise program, and a well-established diagnosis of current CAD as the primary medical condition. Exclusion criteria were presence of any other major medical condition, such as cancer, neurological problems, and known psychotic disorders. Individual clinical data were obtained by reviewing the medical files.

\section{Sedentary group}

The sedentary group consisted of 59 patients consecutively selected from a CAD outpatient clinic in a cardiology center located in the same part of the city. Patients were objectively asked if they were actually physically active and only those with a negative answer to this question were included in the study. Although these patients' level of physical activity or aerobic fitness was not directly or formally evaluated, they will be considered here as the control group for this variable. The inclusion and exclusion criteria for this group were the same as applied to the exercise group, except for the issue of being regularly exercising.

\section{Procedure}

Patients in both groups were interviewed to identify psychiatric disorders and they filled out a questionnaire to measure cardiac anxiety. After agreeing to participate and giving written informed consent, all patients were interviewed by the same trained researcher (A.S.) with the Mini-International Neuropsychiatric Interview (MINI) (10) version 5.0. (14), a short structured interview designed to explore each of the necessary criteria for the main diagnoses of DSM-IV, Axis I. Psychiatric disorders were assessed in terms of present and lifetime prevalence (when possible with the MINI) and in terms of frequency. Information regarding current tobacco use was also collected.

Later, subjects were requested to fill out the validated Brazilian version (15) of the Cardiac Anxiety Questionnaire (CAQ). The CAQ is an 18-item instrument designed to evaluate how threatening the patient considers the experienced cardiac symptoms (11).

\section{Statistical analysis}

To assess differences in the prevalence of psychiatric disorders (PD) and in cardiac anxiety scores between the exercise and sedentary groups, inferential analysis was performed using the software Statistical Packages for Social Sciences (SPSS version 13). Continuous variables were compared by the $t$-test and categorical variables were compared by the Fisher exact test. Cardiac anxiety (CA) scores were also analyzed in association with participation in the exercise program or the sedentary condition using Pearson's correlation. The potential participation of exercise in reducing CAscores was derived using a linear regression model. Last, the demographic and clinical conditions of patients who did or did not return the questionnaires were also compared by the Fisher exact test in order to verify the existence of possible bias. A level of 0.05 was established for statistical significance. The study protocol was approved by the Universidade Federal do Rio de Janeiro and Instituto Estadual de Cardiologia Aloysio de Castro Research Ethics Committees. 


\section{Results}

\section{Demographic and relevant clinical characteristics of the sample}

Exercise group. Patients were 51 men (85\%) and 9 women (15\%) aged 46 to 89 years $(69 \pm 9.7$; mean \pm SD). Most of the patients attended the sessions three times a week (87\%), while 13\% attended 4-6 times a week. Average time of participation in the program was 19.5 months $(S D=11.1)$. Fifty percent $(N=30)$ had a history of at least one acute myocardial infarction (AMI) and 60\% ( $\mathrm{N}=36$ ) were also diagnosed as having arterial hypertension. Only four $(7 \%)$ patients currently smoked. Of the 60 patients interviewed with the MINI, $49(82 \%)$ returned the CAQ. The non-responders did not differ from the complete sample in age $(P=0.83)$, gender $(P=0.63)$, prevalence of $P D(P=$ $0.73)$, or in history of previous AMI $(P=1.0)$.

Sedentary group. This group consisted of a total of 59 patients, $23(39 \%)$ men and $36(61 \%)$ women aged 34 to 87 years $(60.7 \pm 10.3$; mean $\pm S D)$. Thirty-one $(53 \%)$ had no history of AMI, as opposed to $28(47 \%)$ patients who had suffered at least one AMI in the past. Fifty-three (90\%) subjects also had hypertension and $6(10 \%)$ currently smoked. Of the 59 patients interviewed with the MINI, the large majority $(50,84 \%)$ returned the $C A Q$. The remaining nine patients did not differ from the complete sample in age $(P=0.08)$, gender $(P=1.0)$, prevalence of $P D(P=1.0)$, or history of AMI $(P=1.0)$.

\section{Prevalence of psychiatric disorders}

In the exercise group, 27 patients (45\%) met the criteria for at least one psychiatric diagnosis, as explored by the MINI, and 13 patients (21\%) presented multiple PD. On the other hand, 48 patients $(81 \%)$ in the sedentary group had one or multiple PD $(P=0.000)$. There was also a comparable higher number of patients from the sedentary group with multiple PD ( $\mathrm{N}=32 ; 54 \%$; $\mathrm{P}=0.001)$.

In the exercise sample, only 3 patients (5\%) were currently seeing a psychiatrist in outpatient clinical treatment, while among the sedentary patients, $2(3 \%)$ reported being seen by a psychiatrist and were currently medicated with antidepressants and mood stabilizers, a difference that was not statistically significant $(P=0.66)$. Nevertheless, in both settings, patients reported using benzodiazepines on an irregular basis, whenever anxious, stressed of having sleep difficulties, normally prescribed by the cardiologist. Based on patients' self report, none of the patients in the two settings were being regularly submitted to cognitive behavioral therapy (CBT) or other forms of psychotherapy. Also, none of the outpatient clinics where data were collected offered any kind of psychological support.

Among the exercise patients, past depression was found to be the most commonly occurring disorder and was associated in most cases with generalized anxiety disorder (GAD) and agoraphobia. All GAD cases $(N=3,5 \%)$ were subjects with multiple PD. Lifetime prevalence of depressive episode was $15 \%(N=9)$, while only $7 \%(N=4)$ met the criteria for a current major depressive episode. One patient had previously experienced a manic episode and three $(5 \%)$ reported a hypomanic episode in the past. No cases of dysthymia were found. Two patients (4\%) met the criteria for alcohol abuse. No other drug abuse was reported.

Although past depression was also relatively prevalent among sedentary patients ( $N=13,22 \%)$, no significant difference was found between the exercise and sedentary groups $(P=0.344)$. Current depression was the more expressive PD found in these patients ( $N=18,30 \%)$, representing an expressive difference from the four cases $(7 \%)$ identified in the exercise group $(P=0.001)$. A past maniac episode was reported by 1 patient and only 3 reported a history of hypomaniac episodes. In this group, 6 $(10 \%)$ patients were diagnosed with dysthymia according to the MINI. No alcohol or other drug abuse was reported by this sample.

Concerning anxiety manifestations, $42 \%(\mathrm{~N}=25)$ of the subjects in the exercise group reported at least one current anxiety disorder, while among sedentary patients, $33(56 \%)$ presented one or multiple anxiety disorders. No current panic attacks were reported by patients in the exercise group, as opposed to $12 \%$ in the sedentary group. Considering a lifetime timeframe, $6(10 \%)$ participants in the exercise group and $10(17 \%)$ in the sedentary group reported a history of panic attacks $(P=0.40)$.

The most prevalent disorder identified in the exercise group was agoraphobia without panic attacks (18\%, N = $11)$, which was also highly prevalent in the sedentary group $(\mathrm{N}=15,25 \% ; \mathrm{P}=0.260)$. Social phobia was present in $12 \%(\mathrm{~N}=7)$ of the exercisers and in $17 \%(\mathrm{~N}=10)$ of the sedentary patients $(P=0.84)$. Generalized anxiety disorder was about equally present in exercising patients $(\mathrm{N}=3$, $5 \%)$ and in the sedentary group $(N=7,12 \% ; P=0.31)$. Obsessive-compulsive disorder was not diagnosed in any of the patients from the two groups. The frequencies and comparisons of each PD in both groups as established by the MINI are shown in Table 1.

Ninety-nine of the 119 patients $(83 \%)$ returned the CAQ (49 exercisers and 50 sedentary subjects). The mean scores significantly differed between the two groups (37.3 vs 55.8 , respectively; $\mathrm{P}<0.001 ; 95 \% \mathrm{Cl}=13.47$ to 23.55 ), with exercisers presenting lower CA scores than sedentary subjects (see Figure 1).

The severity of cardiovascular disease, defined by a history of AMI, on the other hand, did not influence the $\mathrm{CA}$ scores $(P=0.936,95 \% \mathrm{Cl}=-6.55$ to 6.03$)$; however, when comparing the mean scores of patients with and without a psychiatric diagnosis, regardless of whether the patients were physically active or not, there were significant differences indicating that those who presented at least one psychiatric condition had higher CA scores $(P=0.018$, $95 \% \mathrm{Cl}=-2.14$ to -1.37$)$. 
Table 1. Prevalence of psychiatric disorders in exercising ( $N=$ $60)$ and sedentary $(N=59)$ coronary artery disease patients.

\begin{tabular}{|c|c|c|c|c|}
\hline \multirow[b]{2}{*}{ Variable } & \multicolumn{2}{|c|}{$\begin{array}{l}\text { Exercise } \\
\text { group (\%) }\end{array}$} & \multicolumn{2}{|c|}{$\begin{array}{l}\text { Sedentary } \\
\text { group }(\%)\end{array}$} \\
\hline & Current & Lifetime & Current & Lifetime \\
\hline Depressive episode & 7 & 15 & 30 & 22 \\
\hline Suicide risk & 2 & 0 & 2 & 0 \\
\hline Bipolar disorder & 0 & 7 & 0 & 5 \\
\hline Panic disorder & 0 & 10 & 12 & 17 \\
\hline Agoraphobia & 18 & 0 & 25 & 0 \\
\hline Social phobia & 12 & 0 & 17 & 0 \\
\hline Alcohol abuse & 4 & 0 & 0 & 0 \\
\hline Smoking & 12 & 0 & 10 & 0 \\
\hline Binge eating episodes & 8 & 0 & 10 & 0 \\
\hline $\begin{array}{l}\text { Generalized anxiety } \\
\text { disorder }\end{array}$ & 5 & 0 & 12 & 0 \\
\hline Dysthymia & 0 & 0 & 10 & 0 \\
\hline No psychiatric disorder & 55 & & 19 & \\
\hline
\end{tabular}

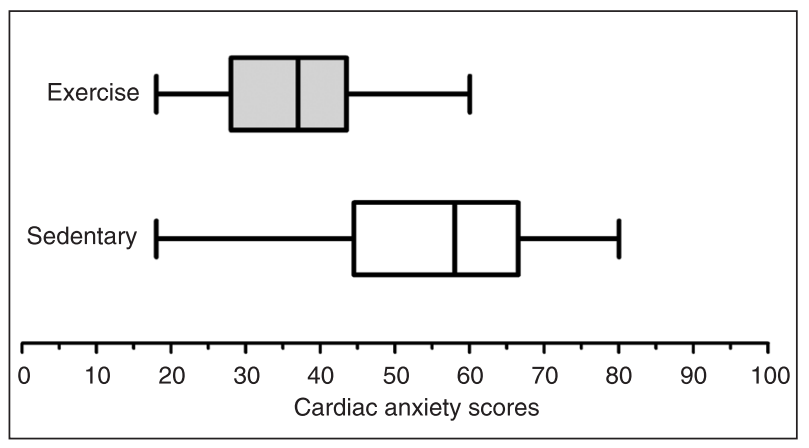

Figure 1. Scores of the Cardiac Anxiety Questionnaire, which was returned by 99 of the 119 patients studied $(83 \% ; 49$ exercisers and 50 sedentary subjects). The mean scores of exercisers were significantly lower than those of sedentary subjects $(P<0.001$, $95 \% \mathrm{Cl}=13.47$ to $23.55, t$-test).

The correlation between duration of participation in the exercising group and CAscores was also analyzed. Average time of participation in the exercise program was found to be 19.5 months (SD = 23.57 months), with a wide range of variance between participants ( $\min =1 ; \max =168$ months). Given this non-parametric distribution, Spearman's correlation was used to establish the association. A weak and nonsignificant correlation of $r=0.219(P=0.147)$ was found between length of participation and higher CA scores.

There was a significant association between CA scores and presence of psychiatric diagnosis $(r=0.239 ; P=0.018)$. On the other hand, there was a strong inverse correlation coefficient between exercise and CA score $(r=-0.597 ; P$ $=0.000$ ). The increase in the number of psychiatric comorbidities seemed to be directly associated with an increased
CA score, but the association was weak and not relevant $(r=0.193 ; P=0.057)$.

In a regression model, the regular participation in an exercise program presented a potential determination $\left(R^{2}\right)$ of $35.6 \%$, statistically significant $(F=53.177 ; \beta=-0597 ; P$ $<0.001$ ), in the reduction of CA. By adding the presence or absence of a psychiatric diagnosis to this model, the increase of potential impact was not significant $(P=0.974)$. None of the other variables inserted significantly increased the determination potential.

\section{Discussion}

The results reported here reveal that CAD patients who were regularly attending a medically supervised exercise program presented less current PD, fewer anxiety disorders and depression, lower indices of multiple PDs and lower CA scores. The cross-sectional methods used do not permit to infer causality between a regular exercise pattern and the presence of PD and CA in these patients. Another limitation of our methods is that the use of the DSM categorical criteria in a structured interview (MINI) to identify psychiatric comorbidities does not inform about the severity of the disorders, but only about its presence or absence. Nevertheless, our data provide clear evidence that CAD patients currently attending a regular exercise program seem to be psychologically healthier than physically inactive patients.

Additionally, the $45 \%$ prevalence of PD in exercising patients confirms the current difficulties among health care providers to recognize and treat psychological disorders in CAD patients. This may add to the hypothesis of Roy-Byrne et al. (2) that health care professionals tend to underestimate anxiety in chronically ill patients, as they consider it to be an unimportant response to illness.

The prevalence of a major depressive episode was the most marked difference between the two groups, with current depression being the more expressive PD found among sedentary patients (30\%) compared to only $7 \%$ in the exercise group. Depression is associated with decreased adherence to medications, a triple risk of noncompliance with medical treatment regimens (1) and reduced chances of successful modifications of other cardiac risk factors and adherence to cardiac rehabilitation (16). It is possible that exercise intervention played an independent role in reducing depression in the exercise group. A review published by Dunn et al. (17) showed that numerous studies report a positive effect of exercise of different intensities on depressive symptoms. More recently, two review studies provided substantial data, especially from cardiac rehabilitation and exercise programs, demonstrating the role of physical activity and cardiorespiratory fitness in improving psychological risk factors such as depression, anxiety, hostility, total psychological stress, as well as stress-related mortality $(18,19)$. 
Although depression may also be a barrier to participation in cardiac rehabilitation and exercise programs, cardiologists can help depressed patients overcome this barrier by providing encouragement (1) or referring them to adequate psychological interventions. CBT has been shown to directly reduce depression in CAD patients (20), helping to overcome social isolation and counseling patients on how to adapt to a healthy lifestyle (21) and could probably be a useful tool to motivate and guide patients toward lifestyle modification and adherence to rehabilitation treatment. CBT incorporates techniques such as self-monitoring, problem solving, and mutual goal setting that reinforce the benefits of exercise as well as other essential self-care strategies that improve clinical outcomes in cardiac patients (10). Depression guidelines for the primary care population have documented that treatments with appropriate pharmacological agents and behavioral interventions are similarly effective and their combination has the benefit of lowering relapse rates (1). Janeway (21) also proposed that, since CBT is also a well-documented evidence-based treatment for anxiety disorders, it should be instituted at the beginning of CAD treatment to ensure that patients understand their condition and improve adherence to treatment. Unfortunately, none of the outpatient clinics where data were collected offered any kind of psychological support.

No significant differences were found in psychiatric medication use between groups. However, a limitation of our method was that information about medication and dosage was collected on a self-report basis, which negatively affects the reliability of the data and prevents more in-depth conclusions about the potential effects of psychiatric drugs on the results obtained.

The high association found between depression and GAD was consistent with literature reports. Although GAD can precede depression and eventually develop into depression, preliminary longitudinal findings suggest that the presence of GAD in patients with major depression does not have prognostic significance in terms of psychiatric impairment (22). Conversely, cardiovascular prognosis in GAD and depressed patients seems to be negatively impacted (6), indicating the need for specific assessment for the presence of GAD and adequate treatment.

Herring et al. (5) found that exercise programs using session durations of at least $30 \mathrm{~min}$, and an anxiety report time frame greater than the past week yield large improvements in anxiety symptoms among sedentary patients who have a chronic illness. Sardinha et al. (23) recently reported a panic disorder case with a favorable response to the inclusion of a 12-week exercise program in the context of CBT. In our study, average time of participation in the exercise program was somewhat longer (mean of 19.5 months), indicating that the impact of exercise on anxiety reduction might last when regular exercise is maintained. Perhaps most importantly, anxiety reduction was favorably associated with a nonspecific anxiety-focused exercise intervention since the exercise program studied was designed as adjuvant treatment only for CAD.

Another aspect to be highlighted is the absence of current panic attacks in the exercise group compared to a $12 \%$ rate among sedentary CAD patients. Although panic disorder patients may possibly avoid participating in a regular exercise program, it seems plausible to hypothesize that exercise might have played a role in reducing panic when considering available evidence from the literature (24). Strohle et al. $(25,26)$ demonstrate in various studies that exercise promotes an acute anti-panic effect in healthy as well as in panic disorder patients (27). Potential underlying mechanisms of this association seem to be the serotonin system $(5,25)$ and, as reported more recently, the brainderived neurotrophic factor (28).

Agoraphobia, although responding to different pharmacological drugs (29), seems not to be significantly influenced by exercise training. Considering the existing evidence that exercise tends to reduce anxiety sensitivity (30), this result may seem puzzling. The rationale of the positive impact of physical activity on anxiety sensitivity relies on the assumption that exercising could be seen as an indirect desensitization process, since autonomic alterations triggered by exercising are similar to anxiety symptoms, but elicited in a safe context $(23,30)$. As the agoraphobia criterion present in the MINI does not focus on the anxiety sensitivity cognitions of the patients, "the fear or the fear", but on the avoidance behaviors, we can hypothesize that the reduction of anxiety sensitivity promoted by exercise could not be assessed with our methods. This could be reinforced by the finding that cardiac-anxiety cognitions were significantly reduced among exercisers, showing that when we consider the more specific aspects of the healthrelated anxiety present in agoraphobic patients, exercise seems to play a positive role.

Cardiac anxiety reduction derived from regular exercise attendance could as well be used to prevent the development of agoraphobic and "panic-like" cognitions and consequent avoidance behaviors in CAD patients at the beginning of treatment. It could be expected that patients after being diagnosed with CAD become worried about their health, and particularly their heart. Health-related anxiety seemed to be more strongly associated with the presence of psychiatric comorbidity than the severity of the cardiovascular condition, confirming data previously reported by Sardinha et al. (31) regarding CAD patients. As regular exercise was the factor most strongly associated with reduced CA in these patients, this emphasizes the relevance of considering the prescription of regular exercise for CAD patients, not only for the cardiovascular benefits, but also as a form of secondary prevention of CA and its deleterious effects on adherence to medical treatment, self-care and subsequent prognosis for these patients.

In this respect, CBT and exercise programs could be used jointly to prevent the negative impact of CAD on the 
patient's mental health, improving treatment adherence, functioning and clinical prognosis (24). Interventions designed to address both physical and psychological symptoms may provide the best method for optimizing functioning and enhancing quality of life in CAD patients (10). Aerobic exercise may reduce depressive and anxiety symptoms in addition to improving cardiovascular fitness. Exercise training can be especially useful for patients who prefer non-pharmacologic treatments for their psychological problems (5). The present study provides clinicians with further evidence to recommend exercise training to their patients

\section{References}

1. Lichtman JH, Bigger JT Jr, Blumenthal JA, Frasure-Smith $\mathrm{N}$, Kaufmann PG, Lesperance F, et al. Depression and coronary heart disease: recommendations for screening, referral, and treatment: a science advisory from the American Heart Association Prevention Committee of the Council on Cardiovascular Nursing, Council on Clinical Cardiology, Council on Epidemiology and Prevention, and Interdisciplinary Council on Quality of Care and Outcomes Research: endorsed by the American Psychiatric Association. Circulation 2008; 118: 1768-1775.

2. Roy-Byrne PP, Davidson KW, Kessler RC, Asmundson GJ, Goodwin RD, Kubzansky L, et al. Anxiety disorders and comorbid medical illness. Gen Hosp Psychiatry 2008; 30: 208-225.

3. Lavie CJ, Milani RV. Adverse psychological and coronary risk profiles in young patients with coronary artery disease and benefits of formal cardiac rehabilitation. Arch Intern Med 2006; 166: 1878-1883.

4. Walters K, Rait G, Petersen I, Williams R, Nazareth I. Panic disorder and risk of new onset coronary heart disease, acute myocardial infarction, and cardiac mortality: cohort study using the general practice research database. Eur Heart $J$ 2008; 29: 2981-2988.

5. Herring MP, O'Connor PJ, Dishman RK. The effect of exercise training on anxiety symptoms among patients: a systematic review. Arch Intern Med 2010; 170: 321-331.

6. Frasure-Smith N, Lesperance F. Depression and anxiety as predictors of 2-year cardiac events in patients with stable coronary artery disease. Arch Gen Psychiatry 2008; 65: 6271.

7. Katerndahl D. Panic plaques: panic disorder \& coronary artery disease in patients with chest pain. J Am Board Fam Pract 2004; 17: 114-126.

8. Frasure-Smith N, Lesperance $\mathrm{F}$. Recent evidence linking coronary heart disease and depression. Can J Psychiatry 2006; 51: 730-737.

9. Braddock AE, Abramowitz JS. Listening to hypochondriasis and hearing health anxiety. Expert Rev Neurother 2006; 6: 1307-1312.

10. Gary RA, Dunbar SB, Higgins MK, Musselman DL, Smith $\mathrm{AL}$. Combined exercise and cognitive behavioral therapy improves outcomes in patients with heart failure. J Psychosom Res 2010; 69: 119-131.

11. Eifert GH, Thompson RN, Zvolensky MJ, Edwards K, Frazer NL, Haddad JW, et al. The cardiac anxiety questionnaire: as a means for reducing the psychological burden that can adversely affect the course of cardiovascular disease.

\section{Acknowledgments}

The authors would like to thank the Clínica de Medicina do Exercício (Clinimex) and Instituto Estadual de Cardiologia Aloysio de Castro professionals for facilitating data collection. Research supported by INCT-Translational Medicine (CNPq) and CAPES. development and preliminary validity. Behav Res Ther 2000; 38: 1039-1053.

12. Strohle A. Physical activity, exercise, depression and anxiety disorders. J Neural Transm 2009; 116: 777-784.

13. Harvey SB, Hotopf M, Overland S, Mykletun A. Physical activity and common mental disorders. Br J Psychiatry 2010; 197: 357-364.

14. Amorim P. Mini International Neuropsychiatric Interview (MINI): validação de entrevista breve para diagnóstico de transtornos mentais. Rev Bras Psiquiatr 2000; 22: 106-115.

15. Sardinha A, Nardi AE, Eifert GH. Translation and crosscultural adaptation of the Brazilian version of the Cardiac Anxiety Questionnaire. Rev Psiq Rio Gd Sul 2008; 30: 139149.

16. Glazer KM, Emery CF, Frid DJ, Banyasz RE. Psychological predictors of adherence and outcomes among patients in cardiac rehabilitation. J Cardiopulm Rehabil 2002; 22: 4046.

17. Dunn AL, Trivedi MH, O'Neal HA. Physical activity doseresponse effects on outcomes of depression and anxiety. Med Sci Sports Exerc 2001; 33: S587-S597.

18. Menezes AR, Lavie CJ, Milani RV, O'Keefe J, Lavie TJ. Psychological risk factors and cardiovascular disease: is it all in your head? Postgrad Med 2011; 123: 165-176.

19. Lavie CJ, Milani RV, O'Keefe JH, Lavie TJ. Impact of exercise training on psychological risk factors. Prog Cardiovasc Dis 2011; 53: 464-470.

20. Rieckmann N, Gerin W, Kronish IM, Burg MM, Chaplin WF, Kong G, et al. Course of depressive symptoms and medication adherence after acute coronary syndromes: an electronic medication monitoring study. J Am Coll Cardiol 2006; 48: 2218-2222.

21. Janeway D. An integrated approach to the diagnosis and treatment of anxiety within the practice of cardiology. Cardiol Rev 2009; 17: 36-43.

22. Moffitt TE, Harrington H, Caspi A, Kim-Cohen J, Goldberg $D$, Gregory AM, et al. Depression and generalized anxiety disorder: cumulative and sequential comorbidity in a birth cohort followed prospectively to age 32 years. Arch Gen Psychiatry 2007; 64: 651-660

23. Sardinha A, Araujo CG, Nardi AE. Interval aerobic training as a tool in the cognitive behavioral treatment of panic disorder. J Bras Psiquiatr 2011; 60: 227-230.

24. Sardinha A, Araujo CG, Soares-Filho GL, Nardi AE. Anxiety, panic disorder and coronary artery disease: issues concern- 
ing physical exercise and cognitive behavioral therapy. Expert Rev Cardiovasc Ther 2011; 9: 165-175.

25. Strohle A, Feller C, Onken M, Godemann F, Heinz A, Dimeo F. The acute antipanic activity of aerobic exercise. Am J Psychiatry 2005; 162: 2376-2378.

26. Strohle A, Hofler M, Pfister H, Muller AG, Hoyer J, Wittchen $\mathrm{HU}$, et al. Physical activity and prevalence and incidence of mental disorders in adolescents and young adults. Psychol Med 2007; 37: 1657-1666.

27. Strohle A, Graetz B, Scheel M, Wittmann A, Feller C, Heinz $A$, et al. The acute antipanic and anxiolytic activity of aerobic exercise in patients with panic disorder and healthy control subjects. J Psychiatr Res 2009; 43: 1013-1017.

28. Strohle A, Stoy M, Graetz B, Scheel M, Wittmann A, Gallinat
$\mathrm{J}$, et al. Acute exercise ameliorates reduced brain-derived neurotrophic factor in patients with panic disorder. Psychoneuroendocrinology 2010; 35: 364-368.

29. Nardi AE, Valenca AM, Freire RC, Mochcovitch MD, Amrein $R$, Sardinha $A$, et al. Psychopharmacotherapy of panic disorder: 8-week randomized trial with clonazepam and paroxetine. Braz J Med Biol Res 2011; 44: 366-373.

30. Smits JA, Berry AC, Rosenfield D, Powers MB, Behar E, Otto MW. Reducing anxiety sensitivity with exercise. Depress Anxiety 2008; 25: 689-699.

31. Sardinha A, Araujo CG, Silva ACO, Nardi AE. Prevalence of psychiatric disorders and health-related anxiety in cardiac patients attending a cardiac rehabilitation program. Rev Psiq Clin 2011; 38: 61-65. 